\title{
Linearized Models of Calcium Dynamics: Formal Equivalence to the Cable Equation
}

\author{
Anthony Zador ${ }^{1, a}$ and Christof Koch ${ }^{2}$ \\ 'Neuroscience Program, Yale University School of Medicine, New Haven, Connecticut 06520 and ${ }^{2}$ Computation and \\ Neural Systems Program, California Institute of Technology, Pasadena, California 91125
}

\begin{abstract}
The dynamics of calcium and other diffusible second messengers play an important role in intracellular signaling. We show here the conditions under which nonlinear equations governing the diffusion, extrusion, and buffering of calcium can be linearized. Because the resulting partial differential equation is formally identical to the one-dimensional cable equation, quantities analogous to the input resistance, space constant, and time constant - familiar from the study of passive electrical propagation-can be defined. Using simulated calcium dynamics in an infinite cable and in a dendritic spine as examples, we bound the errors due to the linearization, and show that parameter uncertainty is so large that most nonlinearities can usually be ignored: robust phenomena in the nonlinear model are also present in the linear model.
\end{abstract}

IKey words: computer models, calcium dynamics, calcium imaging, cable theory, information processing, dendrites, spines]

Computer models are gaining importance as a tool for exploring the role of calcium and other diffusible second messengers in intracellular signaling. They have complemented experimental techniques in a wide variety of systems, including the squid giant synapse (Zucker and Stockbridge, 1983; Yamada and Zucker, 1992), the bullfrog sympathetic ganglion (Yamada et al., 1989; Peng and Zucker, 1993), the crayfish neuromuscular junction (Delaney et al., 1991), and hippocampal dendrites and spines (Connor et al., 1988; Regehr et al., 1989; Müller and Connor, 1991; Jaffe et al., 1992; Malenka et al., 1992). Such models permit estimates of calcium dynamics under conditions when these dynamics are difficult or impossible to observe directly with the experimental technology presently available.

In this article we begin with an expression describing calcium concentration as a nonlinear function of three processes-diffusion, buffering, and pumping. We demonstrate that in certain limiting cases of interest, this equation is formally identical to the linear one-dimensional cable equation. Consequently, quantities analogous to the input resistance, the space constant, and the time constant-familiar to most neuroscientists from the

\footnotetext{
Received July 15, 1993; revised Dec. 27, 1993; accepted Jan. 13, 1994.

We thank Knox Chandler for useful discussions about diffusible buffers, Mike Hines for providing support for NEURON, and Zach Mainen for a careful reading of the manuscript. This research was funded by grants from ONR and the NIMH Center for Neuroscience.

Correspondence should be addressed to Prof. Christof Koch, Division of Biology, 216-76, California Institute of Technology, Pasadena, CA 91125.

Present address: Salk Institute, 10010 North Torrey Pines Road, La Jolla, CA 92037.

Copyright (C) 1994 Society for Neuroscience $0270-6474 / 94 / 144705-11 \$ 05.00 / 0$
}

study of passive electrical propagation - can be simply defined for the reaction-diffusion equation. These quantities enhance our intuitive understanding of calcium dynamics by drawing upon experience from electrical cable theory.

\section{Materials and Methods}

We simulated two nonlinear models of calcium dynamics to illustrate the validity of the linearization. The two models differed primarily in geometry. The first was an infinite cylinder of radius $a=0.5 \mu \mathrm{m}$. The second was a spine consisting of two cylinders: a head of radius 0.25 $\mu \mathrm{m}$ and length $0.3 \mu \mathrm{m}$, connected to a neck of radius $0.05 \mu \mathrm{m}$ and length $1 \mu \mathrm{m}$ (Zador et al., 1990). The spine neck was connected to a large dendritic compartment in which the calcium concentration was clamped either at rest $(0.05 \mu \mathrm{m}$; see Fig. 5) or at some higher value (see Fig. 6). As indicated in the text, calcium stimuli consisted of either a step to some magnitude or a double-exponential waveform.

The parameters governing nonlinear calcium dynamics were the same in both models (see Table 3 ), with the exception that only the highaffinity pump was included in the infinite cable. The spine parameters were the same as those used in the report by Zador et al. (1990), with the difference that in the original model we considered a buffer with four binding sites, while for simplicity in the present model the buffer had only one site. The linearized model corresponding to each nonlinear model was obtained by calculating the membrane parameters $R_{m}, C_{m}$, and $R_{i}$ as described in Equation 15 .

Both the linearized (Eq. 12) and nonlinear (Eq. 3) equations were solved numerically using NEURON, kindly made available by $M$. Hines (Hines, 1989). NEURON is a software package specifically designed for detailed biophysical neuron simulations. All simulations were performed on a Sun Microsystems SPARC IPX workstation. The linear approximations were implemented using the standard built-in code for passive electrical dynamics and setting the membrane parameters $R_{m}$, $C_{m}$, and $R_{i}$ to the appropriate values given by Equation 15. Because NEURON uses a semi-implicit scheme, stable and second-order accurate in $\Delta x$ for all values of the discrete time step $\Delta t$, the linearized equations could be solved very rapidly.

The fully nonlinear model was a reimplementation in NEURON of the original (7ador et al., 1990) model. The pump and buffer were incorporated as compiled "membrane mechanisms," modified from the nmodl files included with standard distributions of NEURON. This implementation made parameter variations very convenient. However, in contrast to the original implementation, the default NEURON implementation solves calcium diffusion with an explicit scheme (forward Euler). This scheme is numerically unstable in the linear case if the discrete time step $\Delta t>2(1+\beta)\left(\Delta x^{2}\right) / P_{m}$, where $\Delta x$ is the spatial discretization, and is only first-order accurate in time (i.e., the error is proportional to $\Delta t$ rather than $\Delta t^{2}$ ). Because of both this instability and inaccuracy, we used a very short time step $(\Delta t=0.5 \mu \mathrm{sec})$ to achieve desired accuracy. Even with this inefficient numerical scheme, typical simulations for the nonlinear equations required less than $1 \mathrm{hr}$.

\section{Analytical Results}

The reaction-diffusion equation

In this section we will build up to a reaction-diffusion equation describing the dynamics of calcium - or any other mobile sub- 
stance-as it diffuses through the interior of a one-dimensional structure in the presence of buffers and surface pumps. Our starting point is the one-dimensional diffusion equation with a source,

$$
\frac{\partial C(x, t)}{\partial t}=D \frac{\partial^{2} C(x, t)}{\partial x^{2}}+\frac{2}{a} i(x, t),
$$

describing the concentration $C(x, t)$ (in $\mu \mathrm{M})$ at time $t$ and position $x$ in response to the applied current density $i(x, t)\left(\mathrm{fA} / \mu \mathrm{m}^{2}\right)$ in terms of the partial derivatives of $C$, the diffusion constant $D$ $\left(\mu \mathrm{m}^{2} / \mathrm{msec}\right)$ of ionic calcium, and the radius $a$ of the cable $(\mu \mathrm{m})$. We assume here that the geometry of the system, for instance a cylindrical apical dendrite of a pyramidal cell, justifies the use of the one-dimensional, rather than the three-dimensional, diffusion equation. In particular, we assume that gradients due to radial diffusion are much less than those due to longitudinal diffusion (see Discussion).

The basic diffusion equation must be augmented to incorporate the physiological handling of calcium in the nerve cell. We consider two additional processes. First, we include a simple calcium extrusion process $P(C)$ that saturates at high $\left[\mathrm{Ca}^{2+}\right.$. Second, we incorporate a diffusible buffer described by the second-order kinetic equation

$$
\mathrm{Ca}^{2+}+B \underset{b}{\stackrel{f}{f}} M
$$

where the rate constants $f$ (in $\left.\mathrm{msec}^{-1}\right)$ and $b\left(\mu \mathrm{M}^{-1} \mathrm{msec}^{-1}\right)$ govern the equilibrium of free calcium $\mathrm{Ca}^{2+}(\mu \mathrm{M})$ and free buffer $B(\mu \mathrm{M})$ with the bound buffer $M(\mu \mathrm{M})$. Incorporating this into the diffusion equation and accounting for the diffusibility of the buffer, we obtain the system of equations

$$
\begin{aligned}
\frac{\partial C}{\partial t} & =D \frac{\partial^{2} C}{\partial x^{2}}-P(C)-f C B+b M+\frac{2}{a} i(x, t), \\
\frac{\partial M}{\partial t} & =D_{h} \frac{\partial^{2} M}{\partial x^{2}}+f C B-b M, \\
B_{T} & =M(x, t)+B(x, t),
\end{aligned}
$$

where $D_{h}\left(\mu \mathrm{m}^{2} / \mathrm{msec}\right)$ is the diffusion constant of both the free and the bound buffer, and $B_{7}$ is the concentration of total buffer. Equation 3 specifies the rate of change in concentration of calcium as a function of diffusion, extrusion, buffer dynamics, and influx. Equation 4 gives the rate of change in bound buffer concentration as a function of buffer diffusion and buffer binding and unbinding. Equation 5 relates the concentrations of free to bound buffer: if, as we have assumed, the free and bound buffers diffuse at the same rate, then in the absence of buffer sources and sinks the total buffer concentration $B_{T}$ is constant.

\section{Linearization}

In this section we show that under certain limiting conditions, in particular, low $\left[\mathrm{Ca}^{2+}\right]$ and fast buffer, the system of nonlinear partial differential equations in Equations 3-5 reverts to a single linear partial differential equation - the cable equation - that has been thoroughly studied in the context of the voltage dynamics in neuronal structures (Jack et al., 1983; Rall, 1989). This reduction allows us to define useful quantities analogous to those familiar from linear cable theory: the membrane time constant $\tau$, the space constant $\lambda$, and the input resistance $R_{\infty}$. In the following section we will use computer simulations to examine the validity of these assumptions in certain cases of biophysical interest.
Pump. The pump we consider has the form

$$
P(C)=\frac{2 P_{m}}{a} \frac{C}{1+C / K_{t}},
$$

where $K_{r}(\mu \mathrm{M})$ is the dissociation constant between the pump and calcium, $2 / a$ is the surface area-to-volume ratio in a cylinder of radius $a$, and the membrane pump parameter $P_{m}(\mu \mathrm{m} / \mathrm{msec})$ is equal to the product of the maximum pump rate (i.e., the maximum number of ions each pump molecule can extrude per unit time) and the pump surface density divided by the pump dissociation constant. The assumption of a pump dissociation constant $\left(K_{p}\right)$, in contrast to the separate forward and reverse buffer binding constants used below, is valid if extrusion is slow relative to diffusion (Haynes and Mandveno, 1987). This pump is saturable, in the sense that if the concentration is high relative to the pump dissociation constant (i.e., $C / K_{v} \gg 1$ ), then $P(C)$ approaches a constant; it behaves as a steady hyperpolarizing current flowing out across the membrane. At the other extreme, if the concentration is low relative to the pump dissociation constant (i.e., $C / K_{b} \ll 1$ ), then extrusion becomes a linear function of $C$; under these conditions $P(C)$ is analogous to the leak term in the cable equation. In summary,

$$
P(C)= \begin{cases}2 P_{m} C / a, & C \ll K_{n}, \\ 2 P_{m} K_{p} / a, & C \gg K_{p} .\end{cases}
$$

Buffer. Under suitable assumptions a buffer can also be incorporated into the linearized equation. First we consider a nondiffusible second-order buffer whose binding kinetics are much faster than the diffusion of calcium. We then extend this approximation to diffusible buffers. In both cases we assume that the kinetics of buffering are much faster than the diffusion of calcium, that is, that Equations 3 and 4 describe a singularly perturbed system (Keener, 1988). While the results in the following paragraph can be obtained rigorously using the methods described in Keener (1988), we opt here for a more intuitive presentation that retains the flavor of the derivation but omits some of the technical complications.

Beginning with a nondiffusible buffer, that is, $D_{b}=0$ in Equation 4, we solve for $M(t)$ while holding $C$ constant to obtain

$$
M(t)=M_{\infty}+\left(M_{0}-M_{\infty}\right) e^{-t / r t},
$$

where $M_{0}$ is the initial concentration of bound buffer, $\tau_{b}=(b+$ $f()^{-1}$ is the buffer time constant, and $M_{\infty}$ is the steady-state buffer concentration given by $B_{7} f C \tau_{i}$. If we assume that the buffer reaches equilibrium much faster than the diffusional processes, we can approximate Equation 4 with a quasi steady-state expression. Thus, near equilibrium $\left(t \gg \tau_{b}\right.$, i.e., $\left.\partial M / \partial t \approx 0\right)$, the concentration of bound buffer can be approximated by

$$
M_{\infty}=B_{T} f C_{\tau_{b}}=\frac{B_{T} C}{K_{c l}+C}
$$

where we have defined the buffer dissociation constant $K_{d}=b / f$. Since we are for now neglecting buffer diffusion $\left(D_{b}=0\right)$, we observe that $\partial M / \partial t=f C B-b M$. We can therefore use the chain rule to computc $\partial M / \partial t$,

$$
\frac{\partial M}{\partial t}=\frac{\partial M}{\partial C} \frac{\partial C}{\partial t}=\frac{B_{r} K_{d}}{\left(K_{d}+C\right)^{2}} \frac{\partial C}{\partial t},
$$

and replace the term $-f C B+b M$ in Equation 3 by this de- 
rivative to arrive at

$$
\left(1+\frac{B_{r} K_{d}}{\left(K_{d}+C\right)^{2}}\right) \frac{\partial C}{\partial t}=D \frac{\partial^{2} C}{\partial x^{2}}-P(C) .
$$

Although this equation does not admit any simple closed-form solution, the entire effect of the buffer is captured within the nonlinear term $1+B_{l} K_{d} /\left(K_{d}+C\right)^{2}$, which acts like a nonlinear capacitance. The steady-state solutions of this equation do not depend on buffering.

For both very high and very low calcium concentrations $C$, this capacitive term becomes linear:

$$
(1+\beta) \frac{\partial C}{\partial t}=D \frac{\partial^{2} C}{\partial x^{2}}-P(C)
$$

If the concentration is low relative to the buffer dissociation constant, $C \ll K_{d}$, then the effective capacitance is approximated by a linear term $(1+\beta)=\left(1+B_{r} / K_{d}\right)$, with the dimensionless constant $\beta=B_{T} / K_{d}$. If the concentration is high, $C \gg K_{d}$, then the capacitance reverts to unity, effectively corresponding to $\beta$ $=0$. The approximation is valid if the buffer time constant is much shorter than the overall time constant due to diffusion.

This linearization shows that the effect of a nondiffusible buffer at low concentration is to add a unitless factor $\beta=B_{T} / K_{d}$ to the effective "capacitance." Even in the absence of buffering, that is, for $B_{T}=0$, there is still an effective capacitance of unity; capacitance is an intrinsic part of the diffusion equation. At low concentrations, a nondiffusible and instantaneous buffer will act to slow down diffusion by reducing $D$ to $D /(1+\beta)$ (and by decreasing the action of the pump by the same amount), while at high concentrations it has no effect, since the buffer is mostly saturated at $C \gg K_{d}$.

We now extend the linearized buffer scheme to include the effect of buffer diffusion, still assuming fast buffer kinetics and low calcium concentration (Junge and McLaughlin, 1987; Irving et al., 1990). We again use Equation 8 to describe the equilibrium concentration of bound buffer $M(x, t)$ as a function of the substrate concentration $C(x, t)$ and the total buffer $B_{T}$. Taking the requisite derivatives in the low calcium limit, we obtain

$$
(1+\beta) \frac{\partial C}{\partial t}=\left(D+\beta D_{h}\right) \frac{\partial^{2} C}{\partial x^{2}}-P(C),
$$

where as before we have defined $\beta=B_{T} / K_{d}$. This shows that the effect of a diffusible buffer is simply to augment the substrate diffusion constant by an amount $\beta D_{k}$ : the effective diffusion constant becomes $\mathrm{D}+\beta D_{b}$. The effective diffusion constant increases because there are now two sources of calcium mobility: direct diffusion at a rate governed by the diffusion constant of free calcium $D$, and a kind of "covert" diffusion of bound calcium riding "piggyback" along with the buffer.

\section{Chemical dynamics and the cable equation}

Equation 12 describes the dynamics of calcium at very low or very high (for $\beta=0$ ) concentrations in a one-dimensional cable. The calcium diffuses with a rate controlled by $D$, is extruded at a rate $P(C)$, and is in equilibrium with a mobile buffer. We now consider the low-concentration limit of this equation $\left(\beta=B_{T} /\right.$ $K_{d l}$ ), for which the extrusion rate is a linear function of the concentration and the radius, $P(C)=2 P_{m} C / a$ (Eq. 7$)$, in the presence of an externally applied point source current term $I(x, t)$. Multiplying through by $a / 2$, we can rewrite Equation 12 as

$$
\begin{aligned}
& \frac{a(1+\beta)}{2} \frac{\partial C(x, t)}{\partial t} \\
& \quad=\frac{a\left(D+\beta D_{t,}\right)}{2} \frac{\partial^{2} C(x, t)}{\partial x^{2}}-P_{m} C(x, t)+K_{\infty} P_{m} I(x, t)
\end{aligned}
$$

Here we have introduced $K_{\infty}$ as the constant of proportionality between a stationary current and the resultant sustained increase in concentration at the point of injection; this allows us to use the current $I(x, t)$ (fA) rather than current density $i(x, t)$.

The advantage of this form is that it emphasizes the formal equivalence between the linearized reaction-diffusion system and the linear cable equation. To see this, consider the propagation of voltage along a one-dimensional fiber of radius $a$, axial resistance $R_{i}$, membrane resistivity $R_{m}$, membrane capacitance $C_{m}$, and input resistance $R_{\infty}$, in response to a current $I(x, t)$ (Jack et al., 1983):

$$
C_{m} \frac{\partial V(x, t)}{\partial t}=\frac{a}{2 R_{i}} \frac{\partial^{2} V(x, t)}{\partial x^{2}}-\frac{1}{R_{m}} V(x, t)+\frac{R_{\infty}}{R_{m}} I(x, t)
$$

The two expressions are formally identical, in that the concentration $C$ plays the role of voltage $V$, if the following substitutions are made:

$$
\begin{aligned}
R_{m}^{-1} & \leftrightarrow P_{m} \\
C_{m} & \leftrightarrow a(1+\beta) / 2 \\
R_{\iota}^{-1} & \leftrightarrow\left(D+\beta D_{h}\right) \\
R_{\infty} & \leftrightarrow K_{\infty}
\end{aligned}
$$

In both cases, the current source is $I(x, t)$.

In one-dimensional cable theory, it is convenient to define the space and time constants, $\lambda$ and $\tau$, characterizing the scale of spatial and temporal dynamics, respectively, and the input resistance $R_{\infty}$, which determines the magnitude of the response to a sustained input. We can define analogous terms for our reaction-diffusion system. Using the standard expressions $\lambda=\sqrt{a R_{m} / 2 R_{i}}, \tau=R_{m} C_{m}$, and the input resistance $R_{\infty}=$ $(2 a)^{-3 / 2} \sqrt{R_{m} / R_{i}} / \pi$ for an infinite cable, we have

$$
\begin{aligned}
\tau_{c} & =\frac{a(1+\beta)}{2 P_{m}}, \\
\lambda_{c} & =\sqrt{\frac{a\left(D+\beta D_{b}\right)}{2 P_{m}}}, \\
K_{\infty} & =\frac{(2 a)^{-3 / 2}}{\pi \sqrt{\left(D+\beta D_{h}\right) P_{m}}} .
\end{aligned}
$$

The space constant $\lambda_{c}$ determines the spatial decrement of calcium in an infinite cable in response to a stationary current at $x=0$ as $C_{0}{ }^{{ }^{r-1 / \lambda} \lambda_{c}}$. We can also define the transfer resistance $K_{i,}$ (Carnevale and Johnston, 1982; Koch et al., 1982) as the ratio of the sustained change in concentration at location $x_{j}$ to the sustained current $I_{i}(t)$ at location $x_{i}$ that gave rise to $C\left(x_{i}, t\right)$. If locations $x_{i}$ and $x_{j}$ are a distance $x_{i j}=x_{j}-x_{i}$ apart in an infinite cylinder, we have

$$
K_{i j}=K_{\infty} e^{-x_{i j / \lambda c}}
$$

Note that in this notation the input resistance is the transfer resistance from a point to itself, $K_{i i}=K_{m}$.

Although the reaction-diffusion and cable equations are for- 
Figure 1. Electrical and chemical space and time constants as a function of radius in an infinite cable. The electrical (dashed lines) and chemical (solid lines) space (left) and time (right) constants are plotted as a function of fiber radius. The scaling behavior of the space constants is the same in both systems, but their magnitudes differ. The scaling behavior of the time constants differs in the two systems. The standard parameters were used (see Table 3 ).
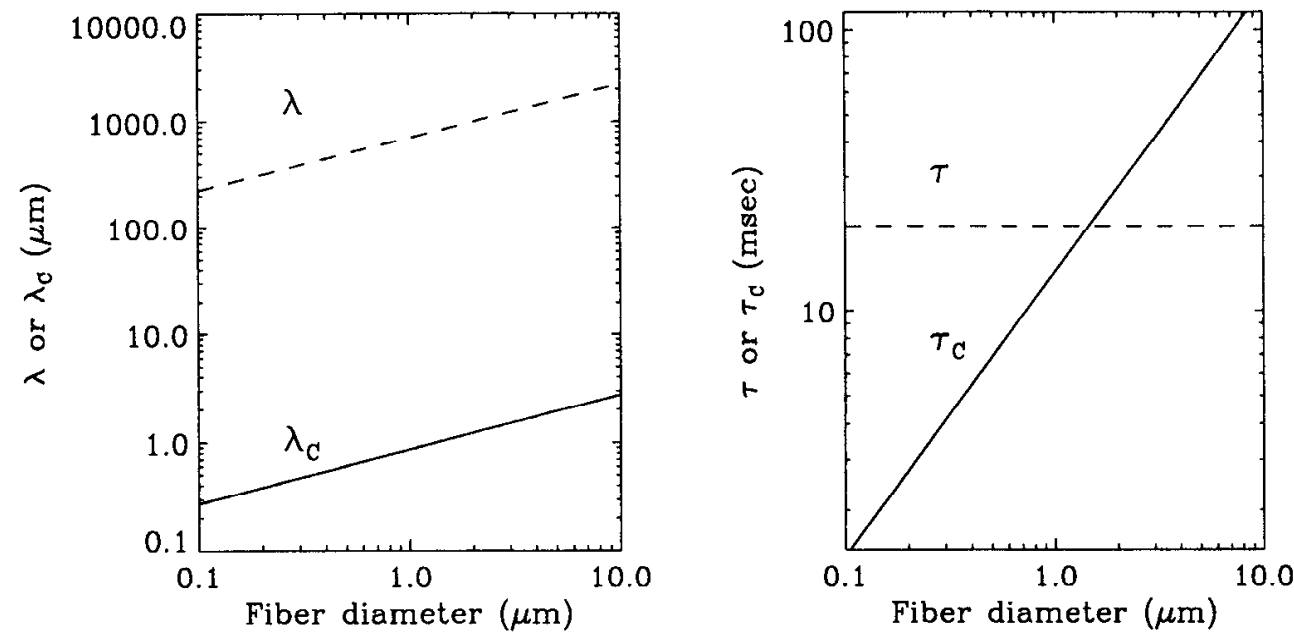

mally equivalent, two differences should be noted. First, transient behavior scales differently in the two systems: the electrical time constant is independent of the fiber radius, while the chemical time constant is a linear function of the radius (Table 1, Fig. 1). The second difference concerns the typical spatial scale of electrical and chemical gradients. In general, the chemical space constant is much shorter than the electrical one:

$$
\lambda_{\mathrm{C}} \ll \lambda
$$

Table 2 compares the electrical and chemical space and time constants for infinite cables with radii $0.05,0.5$, and $5 \mu \mathrm{m}$, assuming our standard chemical and electrical parameters (see Materials and Methods and Table 3). With these parameters the chemical space constant is about three orders of magnitude shorter than the electrical one. Since the chemical space constant scales with the square root of the pump density $\left(\lambda_{c} \propto 1 / \sqrt{P_{m}}\right)$, the pump density would have to be reduced by six orders of magnitude for the two space constants to be similar.

\section{Numerical Simulations}

In order to compare the linearized reaction-diffusion system with the nonlinear system from which it was derived, we examined their behavior in two test cases of interest. First we used the simplest of spatial structures - the infinite cable - to isolate the effect of each simplifying assumption independently of the others. We illustrate how the response of the nonlinear system (Eqs. 3-5) is bounded by the response of the linearized system in the low- and high-concentration limits. This provides an understanding of the kinds of errors introduced by the linearization. Second, we turn to a less idealized case-calcium dynamics in a spine head following activation of NMDA receptors - to examine whether the qualitative conclusions derived from previous extensive study of a nonlinear model (7ador et al., 1990; Koch and Zador, 1993) were preserved in the linear model, and to compare the errors introduced by the linearization with those expected from parameter uncertainty.

\section{Infinite cable}

We begin by examining the effect of nonlinearities due to pump saturation. The time course of calcium concentration $C$ in response to an injected step of current $I_{0}$ depends on whether dynamics are governed by the low- or high-concentration regime. In the low-concentration limit of Equation 12 we can use the standard (see, e.g., Jack et al., 1983; Rall, 1989) closed form solution of the corresponding electrical cable equation:

$$
\begin{aligned}
C(x, t)=K_{\infty} I_{0}[ & e^{-x} \operatorname{erf} \mathcal{C}\left(\frac{X}{2 \sqrt{T}}-\sqrt{T}\right) \\
& \left.-e^{x} \operatorname{erfc}\left(\frac{X}{2 \sqrt{T}}+\sqrt{T}\right)\right],
\end{aligned}
$$

where $\operatorname{erf}()$ is the error function, $\operatorname{erf} c()=1-\operatorname{erf}()$ its complement, and $X=x / \lambda_{c}$ and $T=t / \tau_{c}$ are normalized space and time. In the high-concentration limit the pump saturates, so we revert from the standard cable equation back to the diffusion equation (i.e., with the pump term set to zero). In a single compartment, or for current applied uniformly across the infinite cable, the concentration rises linearly. However, in an infinite cable with a point source, we can find the response to a step injection of current by taking the limit as $P_{m} \rightarrow 0$ of Equation 19:

$$
\begin{aligned}
C(x, t)=\frac{k_{0} I_{0}}{a^{2}} & {\left[\frac{2 \sqrt{t / D(1+\beta)}}{\sqrt{\pi}} e^{x^{2 / 4 D t}}\right.} \\
& \left.-\frac{x}{D} \operatorname{erf}\left(\frac{x \sqrt{1+\beta}}{\sqrt{4 D t}}\right)\right],
\end{aligned}
$$

Table 1. The definition of space and time constants and input resistance for the linear cable equation and the linearized reaction-diffusion equation at low concentrations $C$

\begin{tabular}{llll} 
& Space constant & Time constant & Input resistance \\
\hline Electrical & $\sqrt{a R_{m} / 2 R_{i}}$ & $R_{m} C_{m}$ & $(2 a)^{-3 / 2} \sqrt{R_{m} R_{i}} / \pi$ \\
Chemical & $\sqrt{a\left(D+\beta D_{\mathrm{b}}\right) / 2 P_{m}}$ & $a(1+\beta) / 2 P_{m}$ & $(2 a)^{-3 / 2} / \pi \sqrt{\left(D+\beta D_{b}\right) P_{m}}$
\end{tabular}

Note that the dependence on the fiber radius $a$ of the input resistance and the space constant is the same for both systems, but the dependence of the time constant differs. 
where $k_{0}$ is a proportionality constant. Because neither $\tau_{c}$ nor $\lambda_{c}$. is defined for a cable in the absence of pump $\left(P_{m}=0\right)$, the result is expressed directly in terms of the underlying physical variables (an additional term from Eq. 7 describing the negative current due to the saturated pump has been omitted, since in the high-concentration limit it is negligible compared with the input $I_{0}$ ). Note that the $k_{0} a^{-2}$ term, which replaces the chemical input resistance $K_{\infty}$ in the high-concentration limit, does not have the usual $a^{3 / 2}$ dependence that gives rise to the " $3 / 2$ power law" for matching resistances at branch points (Rall, 1989), but depends instead on $a^{2}$.

One important difference between the two limits is that in the unsaturated pump approximation the concentration approaches some steady-state value $\left(K_{\infty} I_{0}\right.$ at the site of injection), while in the saturated pump limit the concentration increases without bound. In a finite cable the concentration increases without bound whenever the input current exceeds the total extrusion capacity of the cable, which is governed by the pump and the total length of the cable. In an infinite cable the total extrusion capacity is infinite, so that at some distance from the source the concentration drops into the unsaturated regime.

These two linear approximations bound the behavior of the nonlinear pump. For small inputs, the behavior is governed by Equation 19, which describes the unsaturated limit. For large inputs, the behavior is governed by Equation 20. The transition between these two limits is illustrated in Figure 2, where the isolation to the nonlinear system is computed for increasing stimuli at the origin and at four space constants from the origin in an infinite cable using our standard parameters (Table 3 ). For this and subsequent simulations of the infinite cable we adopt
Table 2. Space and time constants and input resistances associated with the chemical and electrical dynamics in an infinite cable of indicated radius for our "standard" model (Table 3)

\begin{tabular}{llclccc}
$a(\mu \mathrm{m})$ & $\lambda_{c}(\mu \mathrm{m})$ & $\tau_{c}(\mathrm{msec})$ & $K_{\infty}(\mu \mathrm{M} / \mathrm{fA})$ & $\lambda(\mu \mathrm{m})$ & $\tau(\mathrm{msec})$ & $R_{\infty}(\mathrm{M} \Omega)$ \\
\hline 0.05 & 0.27 & 1.38 & 0.151 & 224 & 20 & 14,200 \\
0.5 & 0.87 & 13.8 & $4.76 \times 10^{-3}$ & 707 & 20 & 450 \\
5.0 & 2.7 & 138 & $1.51 \times 10^{-4}$ & 2240 & 20 & 14.2
\end{tabular}

Notice that $\lambda_{r} \ll \lambda$ and the different scaling behavior of $\tau_{c}$ and $\tau$. The input resistance is given in equivalent units of $10^{6} \mathrm{M} / \mathrm{A}$ for $K_{\kappa}$ and in $10^{6} \mathrm{~V} / \mathrm{A}$ for $R_{x^{\circ}}$.

the convention that the linearized system is represented by dashed curves, and the response of the nonlinear system by solid curves. The fact that the solid curves fall within the region bounded by the dashed curves shows how the linear equations in the lowand high-concentration limits bound the behavior of the nonlinear system. The concentration has been normalized to give a steady-state of unity at the origin for the small-input case. For the smallest input $I_{0}=10^{-5} \mathrm{nA}$ the simulated curve is superimposed on the predicted curve for low concentration. Larger currents exceed this lower bound but always remain less than the upper bound predicted by the no-pump approximation. Note that the upper bound diverges to infinity as $t \rightarrow \infty$.

Figure 3 shows how buffer saturation affects the validity of the linearization. Because the buffer is assumed to be saturable, the effective capacitance decreases with increasing stimuli. For small stimuli $\left(I_{0}=10^{-4} \mathrm{nA}\right)$ the time course of normalized concentration is well fit by the low-concentration linear approximation. As the stimulus intensity increases, $\tau_{c}$ decreases, so the
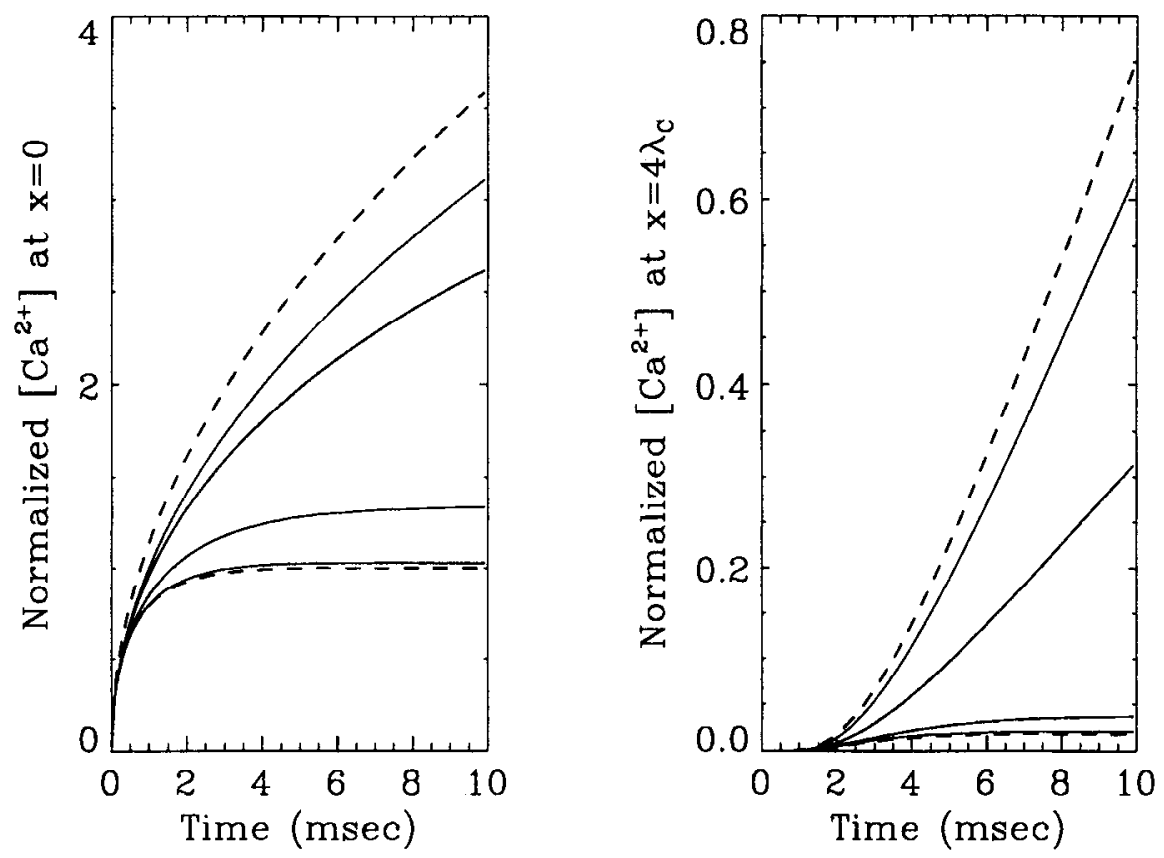

Figure 2. The effect of pump saturation in an infinite cable. The time course of normalized $\left(\left[\mathrm{Ca}^{2+}\right] /\left(K_{\infty} I_{0}\right)\right)$ calcium concentration in response to steady current injection into an infinite one-dimensional cable of radius $a=0.5 \mu \mathrm{m}$ is compared at $x=0$ (left) and $x=4 \lambda_{c}$ ( $($ right). The lower dashed curves show the low-concentration limit (see Table 3 for standard parameters). The upper dashed curves show the high-concentration limit, in which saturation effectively eliminates the effect of the pump (i.e., $P_{m}=0$ ), allowing the concentration to diverge to infinity. The four solid curves show the simulated response of the nonlinear model (Eqs. 3-5) for four different input current magnitudes $\left(I_{0}=10^{-5} \mathrm{nA}, I_{0}=10^{-4} \mathrm{nA}, I_{0}=10^{-3}\right.$ $\left.\mathrm{nA}, I_{0}=10^{-2} \mathrm{nA}\right)$. The lowermost solid curve shows the response to the smallest stimulus, for which the linearized equation with unsaturated pump provides a good approximation. The next three solid curves show the responses to progressively stronger stimuli, illustrating the effect of pump saturation. Note that the linear approximations completely bound the behavior of the full nonlinear system. The buffer has been set to zero $\left(B_{T}=\right.$ 0 ) for these simulations in order to eliminate the confounding influences due to buffer saturation. 

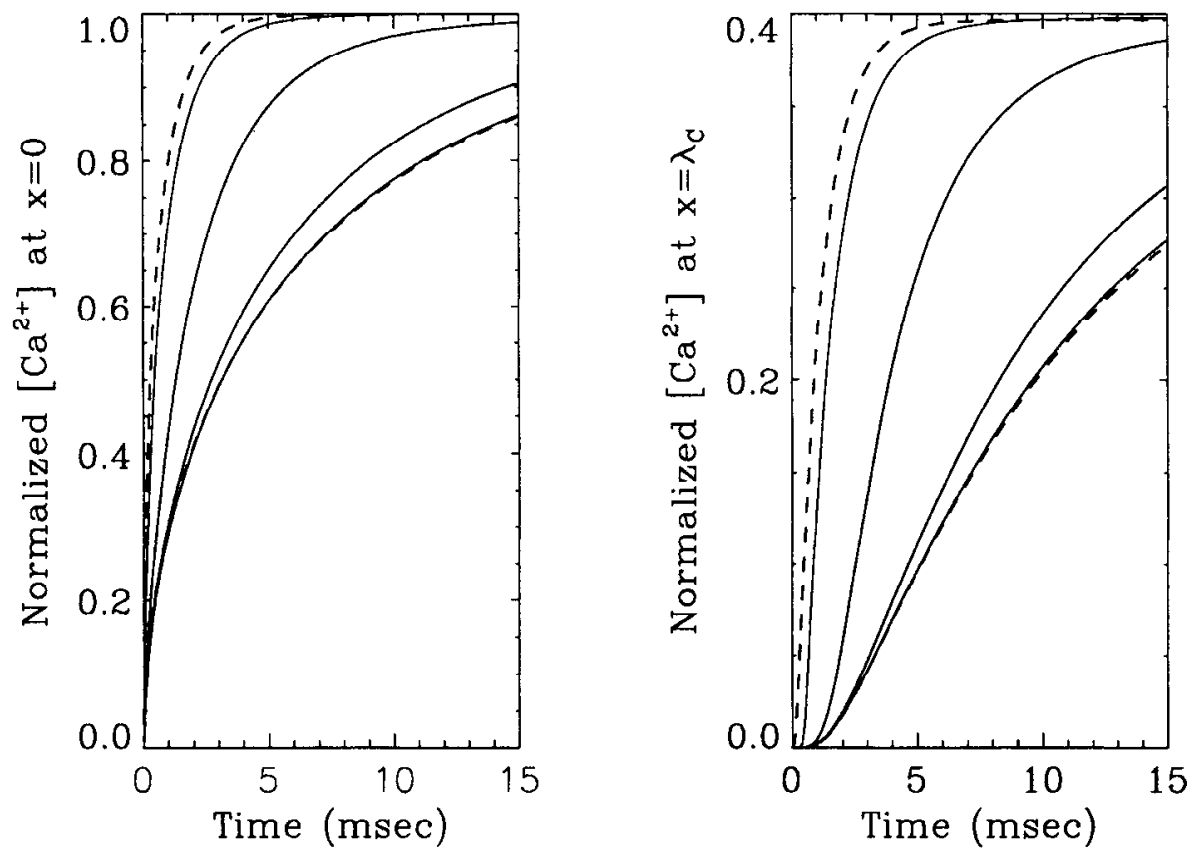

Figure 3. The effect of buffer saturation in an infinite cable. The effect of buffer saturation on the time course of normalized [Ca $\left.{ }^{2+}\right]$ is illustrated at $x=0$ (left) and $x=\lambda_{c}$. (right). The lower dashed curves show the time course for an unsaturated buffer $(\beta=10)$ in the linear approximation, while the upper dashed curves show time course for a fully saturated buffer $(\beta=0)$ in the linear approximation. The four solid curves show the simulated response of the nonlinear model (Eqs. 3-5) for four different input current magnitudes. The lowermost solid curve shows the response to a stimulus that is sufficiently small $\left(I_{0}=10^{-4} \mathrm{nA}\right)$ that the linearized equation with unsaturated buffer provides a good approximation. The next three solid curves show the response to progressively stronger stimuli $\left(I_{0}=10^{-3} \mathrm{nA}, 10^{-2} \mathrm{nA}\right.$, and $\left.10^{-1} \mathrm{nA}\right)$, illustrating the effect of buffer saturation. All curves at a given location eventually reach the same asymptotic value (not shown). In order to isolate the effect of buffer saturation alone, the pump (which has a lower dissociation constant than the buffer and so saturates at a lower value) has been modified to prevent saturation within this range of $\left[\mathrm{Ca}^{2+}\right]$; in addition, the buffer kinetics have been speeded up to ensure buffer equilibrium (see Fig. 4).

concentration rises faster, but the concentration always remains bounded by the $\beta=0$ (no-buffer) limit. The same behavior is observed one space constant away from the stimulus site.

The effect of a buffer not at equilibrium is not conveniently incorporated into the linearized reaction-diffusion equation. In order to test the assumption of fast buffer kinetics underlying the equilibrium simplification (Eq. 8), we compared the simulated time course for buffers with slow and fast kinetics in the low-concentration limit (Fig. 4). The kinetics of calmodulin under physiological conditions have not been determined, so we

Figure 4. Effect of noninstantaneous buffer. The effect of lags in buffer equilibrium on the time course of normalized $\left[\mathrm{Ca}^{2+}\right]$ is illustrated at $x=0($ left $)$ and $x=\lambda_{c}$ (right). The current stimulus $\left(I_{0}=10^{-5} \mathrm{nA}\right)$ is low enough that the low-calcium limit is valid. The dashed curve shows the time course of the linear model assuming an instantaneous unsaturated buffer $(\beta=10)$. The two solid curves illustrate the effect of slow buffer kinetics. For the upper solid curve the standard kinetics were speeded up by three orders of magnitude to guarantee equilibrium. The speeded up forward rate constant was $f=5 \mu \mathrm{M}^{-1} \mathrm{msec}^{-1}$ and the reverse constant was $b=50$ msec $^{-1}$, so using $t_{b}=1 /\left(f\left[\mathrm{Ca}^{+}\right]+b\right)$ we can estimate the buffer time constant at about $20 \mu \mathrm{sec}$. For the lower solid curve the standard kinetics were used, so $\tau_{b}=2 \mathrm{msec}$ was roughly of the same scale as the response. The two curves differ primarily in their behavior during the initial phase. Note that while the noninstantaneous buffer does not constitute a nonlinearity like the saturations considered earlier, it does cause a deviation from the cable approximation.
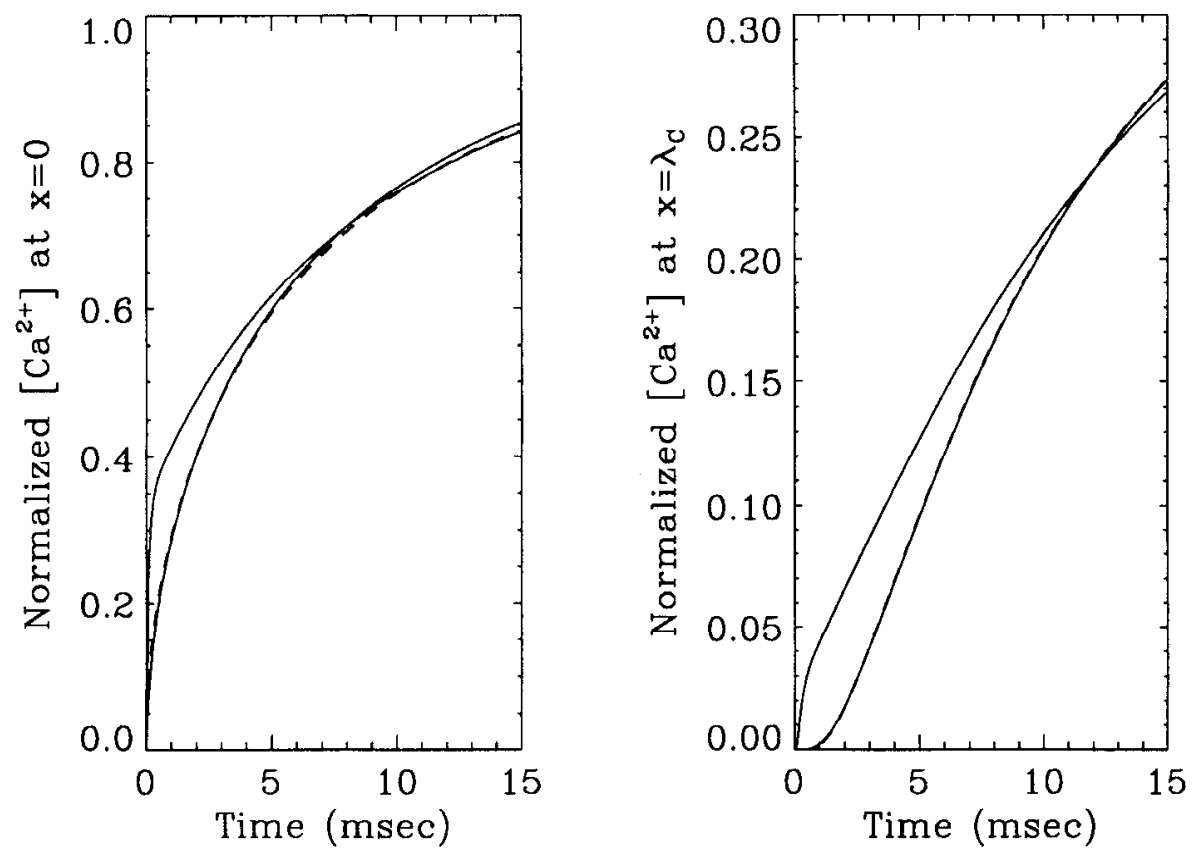
used values derived from in vitro studies (Klee and Haiech, 1980; McBurney and Neering, 1987; see Table 3) for the kinetics. As indicated by the previous figure, the analytic form predicts well the time course of the fast buffer. It also provides a relatively good approximation for most of the time course of the slow buffer, with the largest error during the initial equilibration of the buffer. However, the predicted time course provides neither an upper nor a lower bound for the simulated curve: the curves cross. This crossing does not depend on diffusion, and can in fact be observed for calcium injection into a single compartment with a noninstantaneous buffer. It results from the interaction of two time constants of similar magnitude resulting from a pair of coupled differential equations. If the time scale of buffer equilibration is close to that of diffusion, techniques are available for a more careful analysis of the early time course (Keener, 1988).

We did not simulate the diffusible buffer. We note, however, that the effect of a diffusible buffer can be surprisingly large. Using $D_{b}=0.13$ as the diffusion constant for calmodulin and $\beta=10$, we calculate the effective diffusion constant as $D+\beta D_{h}$ $=0.6+1.3=1.9$ or about three times the usual constant. From Table 1 we see that this leads to a $\sqrt{3}=1.7$-fold increase in the space constant $\lambda$, and a decrease of identical magnitude in the input resistance $K_{\infty}$.

\section{Calcium dynamics in a spine}

While it has only very recently been possible to image calcium dynamics in putative spines of hippocampal pyramidal cells following synaptic input and other manipulations (Guthrie et al., 1991; Müller and Connor, 1991; Jaffe and Brown, 1992), a number of computer simulations of calcium dynamics in spines have appeared (Gamble and Koch, 1987; Holmes, 1990; Holmes and Levy, 1990; Zador et al., 1990; Koch et al., 1992; Koch and Zador, 1993). Following an earlier proposal (Shepherd, 1974), these simulations suggested that spines create a microenvironment for localized changes in the concentrations of ions and second messengers. In order to test the utility of our linearization procedure, we compared a nonlinear model of calcium dynamics in dendritic spines to the corresponding linearized reactiondiffusion equation. Based on the results of the previous section we did expect to observe quantitative differences between the two, especially for short times and/or at high $\left[\mathrm{Ca}^{2+}\right]$. Of real concern is whether qualitative differences result.

We used a nonlinear model of early induction of long-term potentiation (Zador et al., 1990; Koch and Zador, 1993) as the basis for the comparison. Our original model assumed that presynaptic activity activates two postsynaptic conductances located at the spine head: a fast, voltage-independent AMPA conductance and a slower, voltage-dependent NMDA conductance. At resting membrane potentials the NMDA channel is mostly blocked by $\mathrm{Mg}^{2+}$ ions. As the postsynaptic membrane is depolarized, this block is relieved in a voltage-dependent manner, allowing passage of both monovalent cations and calcium ions. With this influx through the channel acting as a point current source, we then simulated the diffusion of calcium ions along the spine neck into the dendrite, their binding to a second-order buffer, and their extrusion into the extracellular space by two different calcium pumps (for more details, see Materials and Methods).

For our present purposes it was sufficient to consider a simplified model corresponding to presynaptic stimulation in conjunction with a postsynaptic voltage clamp; this allowed us to

\begin{tabular}{|c|c|c|}
\hline Symbol & Name & $\begin{array}{l}\text { Default value } \\
\text { and units }\end{array}$ \\
\hline$t$ & Time & msec \\
\hline$x$ & Distance & $\mu \mathrm{m}$ \\
\hline$C$ & {$\left[\mathrm{Ca}^{2+}\right]_{i}$} & $\mu \mathbf{M}$ \\
\hline$V$ & Membrane potential & $\mathrm{mV}$ \\
\hline$D$ & Diffusion constant (calcium) & $0.6 \mu \mathrm{m}^{2} \mathrm{msec}^{-1}$ \\
\hline$I(x, t)$ & Current source & fA \\
\hline$i(x, t)$ & Spatial gradient of current & $\mathrm{fA} / \mu \mathrm{m}$ \\
\hline$M$ & [Bound buffer] & $\mu \mathbf{M}$ \\
\hline$D_{b}$ & Diffusion constant (buffer) & $0.13 \mu \mathrm{m}^{2} \mathrm{msec}^{-1}$ \\
\hline$f$ & Forward buffer rate & $0.05 \mu \mathbf{M}^{-1} \mathrm{msec}^{-1}$ \\
\hline$b$ & Backward buffer rate & $0.5 \mathrm{msec}^{-1}$ \\
\hline$K_{d}$ & Buffer dissociation constant $(b / f)$ & $10 \mu \mathrm{M}$ \\
\hline$B_{T}$ & Total buffer concentration & $100 \mu \mathrm{M}$ \\
\hline$\beta$ & Derived buffer parameter $\left(B_{7} / K_{d}\right)$ & 10 \\
\hline$\tau_{b}$ & Buffer time constant $\left((b+f C)^{-1}\right)$ & msec \\
\hline \multirow[t]{2}{*}{$P_{m}$} & Pump parameter (high affinity) & $0.2 \mu \mathrm{m} \mathrm{msec}-1$ \\
\hline & Low affinity (spine model only) & $0.1 \mu \mathrm{m} \mathrm{msec}^{-1}$ \\
\hline \multirow[t]{2}{*}{$K_{n}$} & Dissociation constant (high affinity) & $0.5 \mu \mathrm{M}$ \\
\hline & Low affinity (spine model only) & $10 \mu \mathrm{M}$ \\
\hline$\tau$ & Electrical time constant & msec \\
\hline$\lambda$ & Electrical space constant & $\mu \mathrm{m}$ \\
\hline$R_{\infty}$ & Electrical input resistance $(V / I)$ & $\mathrm{M} \Omega$ \\
\hline$\lambda_{c}$ & Chemical space constant & $\mu \mathrm{m}$ \\
\hline$\tau_{C}$ & Chemical time constant & msec \\
\hline$K_{\infty}$ & Chemical input resistance $(C / I)$ & $\mu \mathrm{M} \mathrm{fA}^{-1}$ \\
\hline$R_{i}$ & Axial resistance & $100 \Omega \mathrm{cm}$ \\
\hline$R_{m}$ & Membrane resistivity & $20 \mathrm{k} \Omega \mathrm{cm}^{2}$ \\
\hline$C_{m}$ & Membrane capacitance & $1 \mu \mathrm{F} \mathrm{cm}^{-2}$ \\
\hline$a$ & Radius & $\mu \mathrm{m}$ \\
\hline
\end{tabular}

eliminate the details of the voltage dependence of the NMDA receptor channel by considering an injected subsynaptic calcium current $I_{\mathrm{Ca}}(t)$. The time course of this current was given by

$$
I_{\mathrm{Ca}_{2}}(t)=I_{0}\left(e^{-t / \lambda_{1}}-e^{-t / \lambda_{2}}\right) .
$$

This double-exponential expression mimics the time course of the NMDA-mediated synaptic current, with $I_{v}$ governing the peak current and $\tau_{1}=80 \mathrm{msec}$ and $\tau_{2}=3 \mathrm{msec}$ governing the time course (Zador et al., 1990). We compared a small stimulus of $I_{0}=10 \mathrm{fA}$ to a fivefold larger stimulus $I_{0}=50 \mathrm{fA}$; with the values of $\tau_{1}$ and $\tau_{2}$ given, these correspond to peak currents of $8.5 \mathrm{fA}$ and $42 \mathrm{fA}$, respectively.

Figure 5 compares the time courses of $\left[\mathrm{Ca}^{2+}\right]$ at the spine head with the corresponding linearized models. The standard linearized model (lower dashed curves) offers a reasonable fit to the dynamics of the low-intensity stimulus (top panel), but offers only a poor approximation to the higher-intensity stimulus (bottom panel). However, for both stimulus intensities the solution remains bounded by the linear approximation with a 10 -fold lower pump density (upper dashed curve). Because of the tremendous experimental difficulties involved in measuring the densities and affinities of the two calcium pumps in dendrites and spines, this factor of 10 in the pump density represents a very conservative estimate of our uncertainty in this parameter. 
Figure 5. Time course of $\left[\mathrm{Ca}^{2+}\right]$ at the spine head for high-intensity (bottom) and low-intensity (top) synaptic inputs. A calcium current with a slow time course comparable to that of the NMDA receptor channel was applied to the spine head (see text). $I_{0}=10 \mathrm{fA}$ corresponds to a peak current of $8.5 \mathrm{fA}$, while $I_{0}=50 \mathrm{fA}$ corresponds to a peak current of $42.5 \mathrm{fA}$. The solid curves show the simulated time course for the full nonlinear (Eqs. 3-5) model using the standard parameters in Table 3. The dashed curves show that the response of the associated linearized models for the standard parameters (lower dashed curves; $\left.K_{x}=4.2 \times 10^{-2} \mu \mathrm{M} / \mathrm{fA}\right)$ and a 10 -fold reduction in the pump density (upper dashed curves; $\mathrm{K}_{x}=2.8 \times 10$ $\mu \mathrm{M} / \mathrm{fA})$ bound the response of the full model.

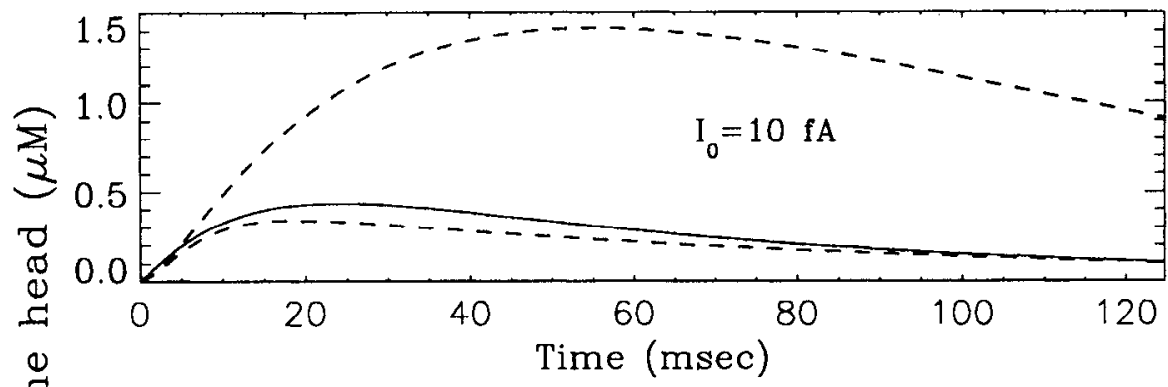

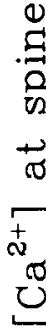

A similar argument can be made for other parameters (e.g., total buffer concentration $B_{T}$ ). This shows how variation of even a single parameter within its range of experimental uncertainty can have a greater effect on the dynamics than does the linearization. In other words, the quantitative error due to linearization is smaller than the error due to uncertainty in the underlying parameters.

Note that during about the first $5 \mathrm{msec}$ the nonlinear model with the standard (slow) buffer kinetics slightly overshoots the linear model because of the lag in buffer equilibration. This same phenomenon was noted for the infinite cable (see Fig. 4). Thus, for very short times the linearized model does not completely bound the solution.

The $10 \mathrm{msec}$ time-to-peak of $I_{\mathrm{Ca}}(t)$ is slow compared to the two chemical time constants -6.9 and $1.4 \mathrm{msec}$-associated with the spine head $(a=0.25 \mu \mathrm{m})$ and the spine neck $(a=0.05$ $\mu \mathrm{m}$ ), respectively (Table 2 ). We can use the parameters of the linear model to estimate directly the peak concentration by multiplying the chemical input resistance at the spine head by the peak current. Using $K_{\infty}=4.2 \times 10^{-2} \mu \mathrm{M} / \mathrm{fA}$, we obtain $C_{\text {peak }}$ $=0.35 \mu \mathrm{M}$ for the low-intensity stimulus and $C_{\text {pcak }}=1.8 \mu \mathrm{M}$ for the higher-intensity stimulus. For the low-intensity stimulus this rough calculation overestimates the actual peak of the linear model by about $25 \%$, but in fact underestimates of the actual peak $(0.44 \mu \mathrm{M})$ for the nonlinear model by only $20 \%$.

That spines amplify calcium currents can be seen by comparing the chemical input resistance at the spine head to the input resistance directly at the dendritic shaft. At the spine head, the input resistance is $4.2 \times 10^{-2} \mu \mathrm{M} / \mathrm{fA}$ for the standard model, while at a dendrite of radius $a=0.5 \mu \mathrm{m}$ it is 10 -fold smaller $\left(K_{\infty}=4.8 \times 10^{-3} \mu \mathrm{M} / \mathrm{fA}\right)$ (Table 2$)$. In other words, a calcium current injected into the spine head leads to roughly a 10 -fold larger increase in calcium concentration than the same current applied at the dendritic shaft.

Another qualitative property noted in the nonlinear model is the ability of spines to isolate the head from sustained changes occurring in the dendritic shaft (Zador et al., 1990; Koch and Zador, 1993). Figure 6 illustrates the decay of calcium along the spine head and neck while the calcium concentration in the dendrite is clamped to $0.6 \mu \mathrm{M}$ or $5.0 \mu \mathrm{M}$. For the standard parameters, the $1 \mu \mathrm{m}$ spine neck has a space constant of $\lambda_{\mathrm{C}}=$ $0.27 \mu \mathrm{m}$ (Table 2). Since the spine neck is about four space constants long, we observe that the steady-state calcium concentration at the spine head decays to approximately $\exp (-4)$, or about $2 \%$ of its dendritic value. The linear model (lower dashed curve) with standard parameters provides an adequate quantitative fit to the lower but not to the higher-concentration clamp experiment. Decreasing $P_{m}$ by 10 (upper dashcd curvc) increases $\lambda_{c}$. by $\sqrt{10}$, and leads to a sustained value of $\left[\mathrm{Ca}^{2+}\right]$ above the curve obtained for the nonlinear model. There is experimental evidence that some spines may not be isolated from their parent dendrite (Guthrie et al., 1991; Müller and Connor, 1991). These results are completely consistent with the linearized model developed here, if they result from actual spine parameters or geometry that lay outside of the narrow range considered here.

\section{Discussion}

Over the last decade, in biophysics as in many other fields, there has been a trend toward more computationally intensive simulations. In the context of calcium dynamics, this has led to an exploration of the complexities of nonlinear diffusional systems in up to three spatial dimensions (Zucker and Stockbridge, 1983; Coss and Perkel, 1985; Simon and Llinás, 1985; Zucker and Fogelson, 1986; Gamble and Koch, 1987; Yamada et al., 1989; Holmes and Levy, 1990; Sala and Hernández-Cruz, 1990; Zador et al., 1990; Meyer and Stryer, 1991; Carnevale and Rosenthal, 1992). One of the main reasons for the growing dominance of the computational approach is the widespread availability of very fast computers, which has opened a wider range of problems to analysis. For many problems the solution cannot be computed analytically but can be found numerically. In other cases, even when a closed form solution does exist it may still be more convenient to find the solution by numerical methods.

The linearized model presented here may therefore appear from a historical perspective a step backward. However, the 


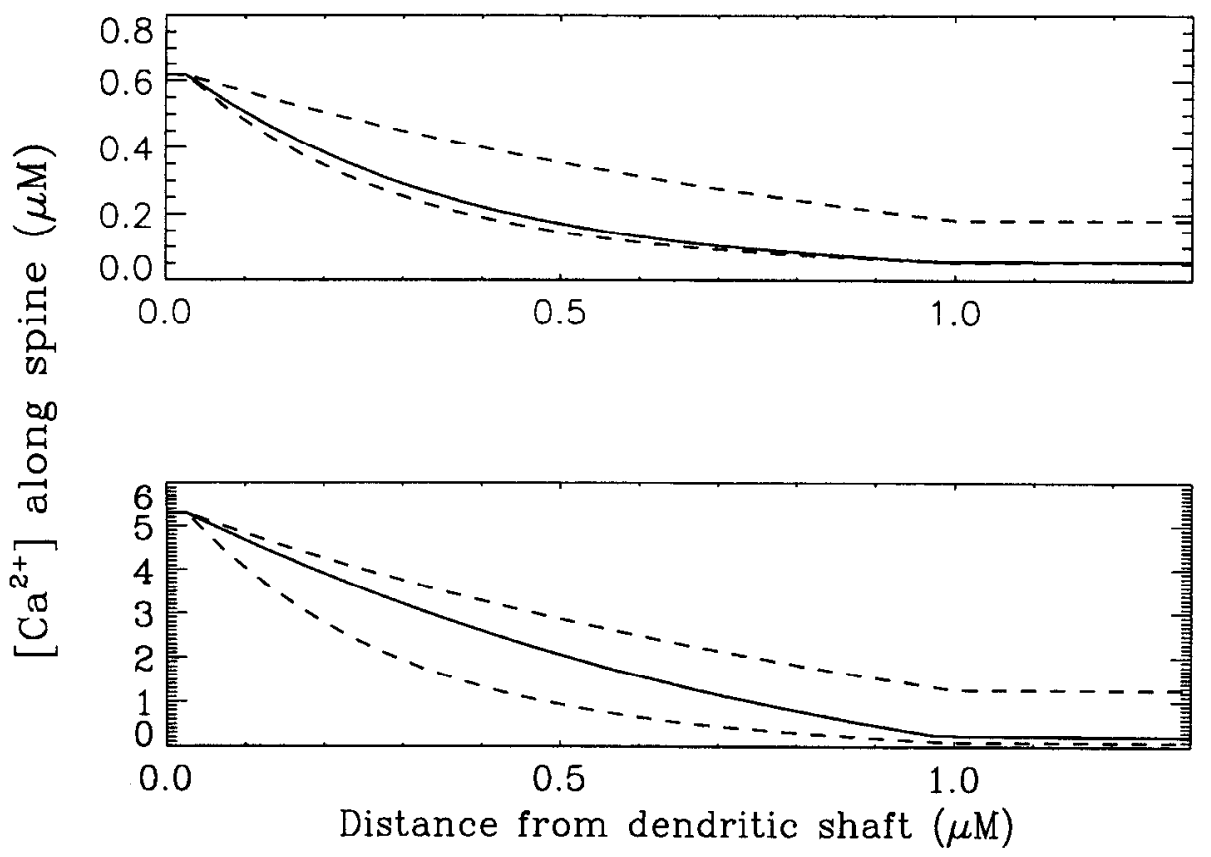

Figure 6. Attenuation of $\left[\mathrm{Ca}^{2+}\right]$ along the spine neck and head while concentration in the dendrite (at the origin of the graph) is clamped either to $0.6 \mu \mathrm{M}$ (top) or to $5.0 \mu \mathrm{M}$ (bottom). The neck extends from $0 \mu \mathrm{m}$ to $1.0 \mu \mathrm{m}$; the decay of $\left[\mathrm{Ca}^{2+}\right]$ along the head $(1 \mu \mathrm{m}$ to 1.3 $\mu \mathrm{m})$ is much slower. The solid curves show the spatial decay for the nonlinear model (Eqs. 3-5). The dashed curves illustrate that the response of the linearized model for the standard (lower curve; $\lambda_{c}=0.27 \mu \mathrm{m}$ ) and 10-fold reduced (upper curve, $\lambda_{c}=0.85 \mu \mathrm{m}$ ) pump densities completely bound the response of the full model. motivation for developing the linearized model was not to make computation faster or more efficient, but rather to provide a framework for understanding the more complex behavior of nonlinear dynamics. In a typical nonlinear model with several dozen free parameters, it is often very difficult to discern which parameters are critical, and what effects they have; this process usually requires extensive trial and error. Further, even once extensive familiarity has been gained with the behavior of a nonlinear model in some parameter regime, it is often difficult to assess the range of validity of the results, or to offer more than anecdotal evidence of the results. The linearized model provides a convenient set of conceptual parameters $\left(K_{\infty}, \tau_{C}, \lambda_{c}\right)$ that are much easier to manipulate and comprehend, and because of the formal correspondence with the well-studied electrical cable equation, we can draw upon the tremendous experience that has accumulated.

The linearization also addresses a too often neglected question: which nonlinearities are required to obtain the qualitative behavior reported? As we have illustrated, it is often precisely the qualitative features of the nonlinear model-that is, those that are robust to large variations in the free parameters, within the bounds of experimental uncertainty-that are preserved within the linear model. In fact, many of the nonlinearities that characterize calcium dynamics tend to be monotonic-typically saturation phenomena-that do not cause the qualitative behavior to deviate in any surprising way from the linear.

\section{Validity of linearized reaction-diffusion equation}

We are primarily concerned here with the dynamics of calcium in dendritic cables. The length of these structures is typically one or two orders of magnitudes larger than their radius, justifying the dimensional reduction of the three-dimensional diffusion equation to a one-dimensional one. Rall (1969) compared the membrane potential derived as the solution of Laplace's equation in three-dimensional cylindrical coordinates with onedimensional cable theory, concluding that the radial contributions of the membrane potential of a dendrite or axon decay
$10^{4}$ times faster than the components of the membrane potential along the axis (see also Eisenberg and Johnson, 1970). A similar analysis for the diffusion equation shows that the one-dimensional approximation is valid as long as (1) the radius is shorter than the length, $a \ll 1$; and (2) the equilibration times for buffering and radial diffusion are short compared to longitudinal diffusion. These conditions may be violated in some situations of physiological interest, such as buffered diffusion over a few fractions of a micron or within fractions of a millisecond (Simon and Llinás, 1985; Zucker and Fogelson, 1986; Roberts, 1993) and "domain" models for $\mathrm{Ca}^{2+}$ inactivation of $\mathrm{Ca}^{2+}$ channels (Sherman et al., 1990; Imredy and Yue, 1992). At these spatial and temporal scales inhomogeneities in the radial direction will certainly arise. The analysis will be further complicated if there are variations in parameters such as buffer concentration along the radial axis; under such conditions some average value of the parameter must be used. Nevertheless, the simple reactiondiffusion equation derived here is useful for analyzing calcium dynamics in dendrites and spines (Connor et al., 1988; Tank et al., 1988; Guthrie et al., 1991; Regehr and Tank, 1992), and can be extended to include diffusion and buffering in a spherical cell body (Connor et al., 1988; Yamada et al., 1989; Sala and Hernández-Cruz, 1990; Carnevale and Rosenthal, 1992).

There are many conditions under which nonlinearities can not be neglected; we call these essential nonlinearities. For example, in the study of electrical signal propagation it is clear that the nonlinearities of voltage-dependent membrane channels are essential to the generation of action potentials. Similar nonlinear models have been proposed to explain calcium waves and oscillations seen in astrocytes and other cells (Meyer and Stryer, 1991; Berridge, 1993). Multiple cooperative binding steps to calcium buffers, such as calmodulin with its four binding sites, may serve to sharpen the relationship between concentration and certain biochemical reactions, such as autophosphorylation (Lisman, 1985); this sharpening may be thought of as implementing a threshold type of nonlinearity (Koch and Poggio, 1983, 1987). In some cases, the time dependence of an 
essential nonlinearity may be neglected; in such cases, the phenomenon may be modeled as a linear system system followed by a static nonlinearity.

\section{Space- and time-constants of reaction-diffusion equation}

Recognition of its equivalence to the electrical cable equation offers insight into the interpretation of the reaction-diffusion equation (see also Sejnowski and Quian, 1992). The pump $P_{m}$ acts like a membrane conductance $R_{m}{ }^{-1}$, since calcium ions "leak" through the membrane: the greater the pump density, the greater the decrement per unit distance of calcium. The buffer, like a capacitance, acts as a storage device. Specifically, the buffer adds to the capacitance by an amount equal to the total concentration of buffer divided by its affinity, $\beta=B_{T} / K_{d}$. Because all of the calcium that binds to the buffer may ultimately be released into its free state again, the buffer affects only the transient behavior (neglecting the effect of buffer diffusion on the effective diffusion constant $D+\beta D_{b}$ ). Note, however, thatin marked contrast to the electrical cable equation - the buffer does not completely determine the transient behavior: if the buffer concentration is zero, the effective capacitance is unity, while if the membrane capacitance is zero, the result is a purely resistive grid with no transient behavior. Finally, recalling a classical result from statistical physics (see, e.g., Hille, 1992), the diffusion coefficient $D$ is analogous to the inverse of axial resistance, $R_{i}^{-1}$. Just as the axial resistance determines the spread of potential along the longitudinal axis, so the diffusion constant determines the rate of calcium flux along the longitudinal axis.

Equation 13 also allows us to define a space constant $\lambda_{c}$, a time constant $\tau_{C}$, and an input resistance $K_{\infty}$. At least two significant differences exist between $\lambda_{c}$ and $\tau_{c}$ and their electrical analogs (see Tables 1, 2). First, given reasonable values of calcium pump densities (Hille, 1992), the chemical space constant is much greater than its electrical counterpart (Table 2). Thus, while the spatial extent of steady-state voltage gradients in passive dendritic trees is of the same order of magnitude as the tree itself (Rall, 1989), concentration gradients remain much more localized. This results in an important difference between the electrical and chemical behavior of spines (Shepherd, 1974; Harris and Stevens, 1989): experimentally evoked changes in calcium concentration at the dendritic shaft may not be paralleled in the spine (Guthrie et al., 1991) because the chemical space constant may be of the same order of magnitude as the spine neck.

A further difference is that transient behavior scales differently in the two systems. As shown in Table 1, the chemical time constant is proportional to the fiber radius, $\tau_{c} \propto a$, while the electrical time constant $\tau$ is independent of fiber radius. Thus, chemical dynamics are slower in larger cables. The difference between the two systems arises from the nature of the capacitative terms. In electrical dynamics both capacitance and leak are properties of the membrane that scale with surface area. In chemical dynamics the leak due to surface pumps is a membrane property that scales with surface area, while the capacitance is an intrinsic property of the cable that scales with volume. Since it is the ratio of the capacitance and the leak that gives rise to $\tau$ in both cases, the scaling properties differ. Therefore, the time scales of calcium and voltage dynamics are comparable for small cylinders, but can differ greatly for thicker dendrites.

By contrast, both the input resistance $\left(R_{\infty} \propto a^{-3 / 2}, K_{\infty} \propto a^{-3 / 2}\right)$ and the space constant $\left(\lambda \propto \sqrt{a}, \lambda_{c} \propto \sqrt{a}\right)$ scale identically in the two systems. Since the chemical input resistance has the same $a^{3 / 2}$ dependence as the electrical input resistance, it too obeys the " $3 / 2$ power law" (Rall, 1989) for matching impedances at branch points. The definition of a transfer resistance (Eq. 17) allows one to define calcium attenuation coefficient between points $i$ and $j$, that is, the ratio of sustained (or peak) $C$ at location $x_{i}$ to sustained (or peak) $C$ at location $x_{j}$ (Carnevale and Johnston, 1982; Koch et al., 1982). In particular, this allows the "morphoelectrotonic"- or in this case "morphocalcitonic"-transform to be applied to any dendritic tree (Zador et al., 1991), so that the morphology of the tree can be recast in units of calcium attenuation.

We believe that application of these techniques will ultimately help us better understand the role that calcium-or any other diffusible second messenger-plays in information processing in the dendritic trees.

\section{References}

Berridge M (1993) Inositol trisphosphate and calcium signalling. Nature 361:315-325.

Carnevale N, Johnston D (1982) Electrophysiological characterization of remote chemical synapses. J Neurophysiol 47:606-621.

Carnevale NT, Rosenthal S (1992) Kinetics of diffusion in a spherical cell. I. No solute buffering. J Neurosci Methods 41:205-216.

Connor J, Wadman W, Hockberger P, Wong R (1988) Sustained dendritic gradients of $\mathrm{Ca}^{2+}$ induced by excitatory amino acids in $\mathrm{CAl}$ hippocampal neurons. Science 240:649-653.

Coss R, Perkel D (1985) The function of dendritic spines: a review of theoretical issues. Behav Neural Biol 44:151-185.

Delaney K, Tank D, Zucker R (1991) Presynaptic calcium and serotonin-mediated enhancement of transmitter release at crayfish neuromuscular junction. J Neurosci 1 1:2631-2643.

Eisenberg R, Johnson E (1970) Three-dimensional electrical field problems in physiology. Prog Biophys Mol Biol 20:1-65.

Gamble E, Koch C (1987) The dynamics of free calcium in dendritic spines in response to repetive synaptic input. Science 236:1311-1315.

Guthrie P, Segal M, Kater SB (1991) Independent regulation of calcium revealed by imaging dendritic spines. Nature $354: 76-80$.

Harris K, Stevens J (1989) Dendritic spines of CAl pyramical cells in the rat hippocampus: serial electron microscopy with reference to their biophysical characteristics. J Neurosci 9:2982-2997.

Haynes D, Mandveno A (1987) Computer modeling of $\mathrm{Ca}^{2+}$ pump function of $\mathrm{Ca}^{2+}-\mathrm{Mg}^{2+}$-ATPase of sarcoplasmic reticulum. Physiol Rev 67:244-284.

Hille B (1992) Ionic channels of excitable membranes, 2d ed. Sunderland, MA: Sinauer.

Hines M (1989) A program for simulation of nerve equations with branching geometries. Int J Biomed Comput 24:55-68.

Holmes W (1990) Is the function of dendritic spines to concentrate calcium? Brain Res 519:338-342.

Holmes W, Levy W(1990) Insights into associative long-term potentiation from computational models of NMDA receptor-mediated calcium influx and intraccllular calcium concentration changes. J Ncurophysiol 63:1148-1168.

Imredy J, Yue D (1992) Submicroscopic $\mathrm{Ca}^{+2}$ diffusion mediates inhibitory coupling between individual $\mathrm{Ca}^{+2}$ channels. Neuron 9:197207.

Irving M, Maylie J, Sizto N, Chandler W (1990) Intracellular diffusion in the presence of mobile buffers: application to proton movement in muscle. Biophys J 57:717-721.

Jack J, Noble A, Tsien R (1983) Electrical current flow in excitable membranes, $2 \mathrm{~d}$ ed. New York: Oxford UP.

Jaffe D, Brown T (1992) Confocal imaging of thorny excrescences on hippocampal pyramidal neurons. Soc Neurosci Abstr 18.

Jaffe D, Johnston D, Lasser-Ross N, Lisman J, Miyakawa H, Ross W (1992) The spread of $\mathrm{Na}$ spikes determines the pattern of dendritic $\mathrm{Ca}^{+?}$ entry into hippocampal neurons. Naturc 21:244-246.

Junge W, McLaughlin S (1987) The role of fixed and mobile buffers in the kinetics of proton movement. Biochim Biophys Acta 890:1-5.

Keener J (1988) Principles of applied mathematics: transformation and approximation. Reading, MA: Addison-Wesley. 
Klee C, Haiech J (1980) Concerted role of calmodulin and calcineurin in calcium regulation. Ann NY Acad Sci 356:43-54.

Koch C, Poggio T (1983) Electrical properties of dendritic spines. Trends Neurosci 6:1-4.

Koch C. Poggio T (1987) Biophysics of computation: neurons, synapses, and membranes. In: Synaptic function (Edelman G, Gall W, Cowan W, eds), pp 637-683. New York: Wiley.

Koch C. Zador A (1993) The function of dendritic spines: devices subserving biochemical rather than electrical compartmentalization. J Neurosci 13:413-422.

Koch C, Poggio T. Torre V (1982) Retinal ganglion cells: a functional interpretation of dendritic morphology. Philos Trans R Soc Lond [Biol] 298:227-264.

Koch C, Zador A. Brown T (1992) Dendritic spines: convergence of theory and experiment. Science 256:973-974.

Lisman J (1985) A mechanism for memory storage insensitive to molecular turnover: a bistable autophosphorylating kinase. Proc Natl Acad Sci USA 82:3055-3057.

Malenka R, Lancaster B, Zucker R (1992) Temporal limits on the rise in postsynaptic calcium required for the induction of long-term potentiation. Neuron 9:121-128.

McBurney R, Neering I (1987) Neuronal calcium homeostasis. Trends Neurosci 10:164-169.

Meyer T, Stryer L (1991) Calcium spiking. Annu Rev Biophys Biophys Chem 20:153-174.

Müller W, Connor J (1991) Dendritic spines as individual neuronal compartments for synaptic $\mathrm{Ca}^{2}$ ' responses. Nature 354:73-76.

Peng Y, Zucker R (1993) Release of LHRH is linearly related to the time integral of presynaptic $\mathrm{Ca}^{+2}$ elevation above a threshold level in bulffrog sympathetic ganglia. Neuron 10:465-473.

Rall W (1969) Distribution of potential in cylindrical coordinates and time constants for a membrane cylinder. Biophys J 9:1509-1541.

Rall W (1989) Cable theory for dendritic neurons. In: Methods in neuronal modeling (Koch C, Segev I, eds), pp 9-62. Cambridge, MA: MIT Press.

Regehr W. Tank D (1992) Calcium concentration dynamics produced by synaptic activation of cal hippocampal pyramidal cells. J Neurosci $12: 4202-4203$
Regehr W, Connor J, Tank D (1989) Optical imaging of calcium accumulation in hippocampal pyramidal cells during synaptic activation. Nature 341:533-536.

Roberts W (1993) Spatial calcium buffering in saccular hair cells. Nature 363:74-76.

Sala F, Hernández-Cruz A (1990) Calcium diffusion modelling in a spherical neuron. Biophys J 57:313-324.

Sejnowski T, Quian N (1992) Synaptic integration by electrodiffusion in dendritic spines. In: Single neuron computation (McKenna T, Davis J, Zornetzer S, eds), pp 117-139. New York: Academic.

Shepherd G (1974) The synaptic organization of the brain, lst ed. New York: Oxford UP.

Sherman A, Keizer J, Rinzel J (1990) Domain model Ca+2-inactivation of $\mathrm{Ca}^{+2}$ channels at low channel density. Biophys J 58:985995.

Simon S. Llinás R (1985) Compartmentalization of submembrane calcium activity during calcium influx and its significance in transmitter release. Biophys J 48:559-569.

Tank D, Sugimori M. Connor J, Llinas R (1988) Spatially resolved calcium dynamics of mammalian Purkinje cells in cerebellar slice. Science 242:773-777.

Yamada W, Zucker R (1992) Time course of transmitter release calculated from simulations of a calcium diffusion model. Biophys J 61 : $671-682$

Yamada W, Koch C, Adams P (1989) Multiple channels and calcium dynamics. In: Methods in neuronal modeling (Koch C, Segev I, eds), pp 97-133. Cambridge, MA: MIT Press.

Zador A, Koch C, Brown T (1990) Biophysical model of a Hebbian synapse. Proc Natl Acad Sci USA 87:6718-6722.

Zador A, Claiborne B, Brown T (1991) Electrotonic transforms of hippocampal neurons. Soc Neurosci Abstr 17:1515.

Zucker R, Fogelson A (1986) Relationship between transmitter release and presynaptic calcium influx when calcium enters through discrete channels. Proc Natl Acad Sci USA 83:3032-3036.

Zucker R, Stockbridge N (1983) Presynaptic calcium diffusion and the time courses of transmitter release and synaptic facilitation at the squid giant synapse. J Neurosci 3:1236-1269. 